\title{
Avaliações Térmica e Reológica da Matriz Termoplástica PEKK Utilizada em Compósitos Aeronáuticos
}

\author{
Rogério L. Mazur, Edson C. Botelho \\ Departamento de Materiais e Tecnologia, UNESP, Guaratinguetá/SP \\ Michelle L. Costa, Mirabel C. Rezende \\ Divisão de Materiais, Instituto de Aeronáutica e Espaço, CTA
}

\begin{abstract}
Resumo: A matriz termoplástica semicristalina PEKK (poli (éter-cetona-cetona)) tem recebido nos últimos anos uma atenção crescente pelas indústrias aeronáutica e espacial, no processamento de compósitos poliméricos avançados. As características de elevados valores de temperatura de transição vítrea, de resistência mecânica e de módulo de elasticidade, baixa absorção de umidade, excelente resistência a variações de condições climáticas, combinadas a uma relativa baixa massa específica faz do PEKK uma atrativa opção para uma variedade de aplicações, principalmente na área aeronáutica. O objetivo deste trabalho é correlacionar parâmetros térmicos e reológicos do PEKK, por meio das técnicas de calorimetria exploratória diferencial (DSC), termogravimetria (TG) e reológica, no estabelecimento de um ciclo térmico de processamento, a ser utilizado na obtenção de laminados de PEKK com fibras de carbono, pela técnica de moldagem por compressão a quente de compósitos termoplásticos. Os resultados obtidos mostram que o PEKK apresenta um excelente balanço de propriedades, tais como elevada temperatura de transição vítrea $\left(153-156^{\circ} \mathrm{C}\right)$ e moderadas temperaturas de processamento, localizadas entre suas temperaturas de fusão $\left(310-325{ }^{\circ} \mathrm{C}\right)$ e de degradação $\left(352-366^{\circ} \mathrm{C}\right)$, principalmente quando comparado com as matrizes atualmente mais utilizadas no setor aeronáutico: o PEEK (poli (éter-éter-cetona)); o PEI (poli (éter-imida)) e o PPS(poli(sulfona). A partir dos resultados térmicos e reológicos obtidos estabeleceu-se um ciclo térmico a ser utilizado no processamento de laminados PEKK/fibras de carbono pelo uso da moldagem por compressão a quente.
\end{abstract}

Palavras-chave: PEKK, compósitos termoplásticos avançados, análises térmicas, análises reológicas, processamento.

\section{Thermal and Rheological Evaluation of PEKK Thermoplastic Matrix for Aeronautical Application}

Abstract: Semi-crystalline PEKK thermoplastic matrix used in advanced polymeric composites have received a lot of interest for aerospace application in the last years. The higher glass transition temperature, high strength and stiffness, low moisture absorption, excellent environmental resistance combined with its low density make PEKK an attractive option for a variety of applications in aerospace and aeronautical field. The aim of the present work is to analyze the influence of the thermal and rheological parameters of PEKK using the techniques Fourier Transform InfraRed (FT-IR), differential scanning calorimetry (DSC), thermogravimetry (TG) and rheology, in the definition of processing thermal cycle to be utilized with the hot compression molding of thermoplastic composites. The results obtained from the thermal and rheological analyses showed that PEKK has an attractive balance of properties, such as a high transition temperature $\left(\mathrm{T}_{\mathrm{g}}=153-156^{\circ} \mathrm{C}\right)$ and of being able to be processed at a moderate temperature, between the melting $\left(310-325^{\circ} \mathrm{C}\right)$ and degradation $\left(352-366^{\circ} \mathrm{C}\right)$ temperatures, mainly when compared with other polymers, including PEEK (polyether ether ketone), PEI (Polyetherimide) and PPS (polyphenylene sulfide). From these results, it was possible to establish appropriate thermal and rheological parameters to be used in hot compression molding of advanced polymeric laminates.

Keywords: PEKK, advanced thermoplastic composites, thermal analyses, rheological analyses, processing.

\section{Introdução}

Os compósitos poliméricos avançados utilizados em aplicações aeronáuticas e espaciais vêm conquistando cada vez mais uma maior participação no mercado internacional. O rápido desenvolvimento na ciência e tecnologia dos materiais, a necessidade de materiais de elevado desempenho e o desenvolvimento de sofisticados métodos numéricos para análise estrutural, aliados à necessidade de garantia da qualidade, reprodutibilidade e previsibilidade do comportamento sobre a vida da estrutura, têm impulsionado a produção de materiais de elevadas resistência mecânica e rigidez, baixa massa específica e custo competitivo ${ }^{[1]}$.

Atualmente, alguns polímeros termoplásticos de alto desempenho, tais como: PEEK (poli (éter-éter-cetona)), PEI (poliéterimida) e PPS (poli (sulfeto de fenileno)) vêm apresentando uma grande participação no mercado aeronáutico. Embora estes polímeros apresentem excelentes proprie-

Autor para correspondência: Rogério L. Mazur, Departamento de Materiais e Tecnologia, Universidade Estadual Paulista - UNESP, CEP: 12516-410,

Guaratinguetá, SP, Brasil. E-mail: rogermaz@uol.com.br 
dades físicas, mecânicas e térmicas, conforme apresentado na Tabela $1^{[2-4]}$, os rígidos requisitos e o mercado atrelados à indústria aeronáutica motivam a busca por matrizes poliméricas de melhor desempenho e custos mais competitivos. Dentro deste contexto, o PEKK (poli (éter-cetona-cetona)) apresenta um conjunto de propriedades com excelente potencial para aplicações estruturais, quando se deseja alcançar elevada resistência mecânica, baixa massa específica e elevadas temperaturas de serviço, propriedades essas sempre aliadas a um custo competitivo ${ }^{[3-7]}$, tornando-o uma atrativa opção para uso em compósitos poliméricos avançados utilizados em aplicações aeroespaciais.

O PEKK é um polímero termoplástico de alto desempenho, que possui um excelente balanço de propriedades, como alta temperatura de transição vítrea $\left(\mathrm{T}_{\mathrm{g}}=156^{\circ} \mathrm{C}\right)$, elevados valores de resistência mecânica e de rigidez, alta tenacidade à fratura, baixa absorção de umidade e excelente resistência a diversas condições climáticas ${ }^{[3-7]}$.

O PEKK é um polímero que faz parte da família do PAEK (poli (aril-éter-cetona)) que também inclui o PEK (poli (éter-cetona)), o PEKEKK (poli (éter-cetona-étercetona-cetona)) e o mais conhecido PEEK (poli (éter-éter-cetona)). Este último, apesar de mais tempo no mercado, e, conseqüentemente mais bem avaliado, ainda apresenta um custo elevado e dificuldades no seu processamento para a obtenção de peças com propriedades bem definidas, devido à sua elevada temperatura de processamento (382 a $399^{\circ} \mathrm{C}$ ) e a alta viscosidade quando fundido. De uma maneira geral, todos os polímeros mencionados apresentam

Tabela 1. Principais propriedades dos polímeros PEEK, PEI, PPS e PEKK $^{[2-4]}$.

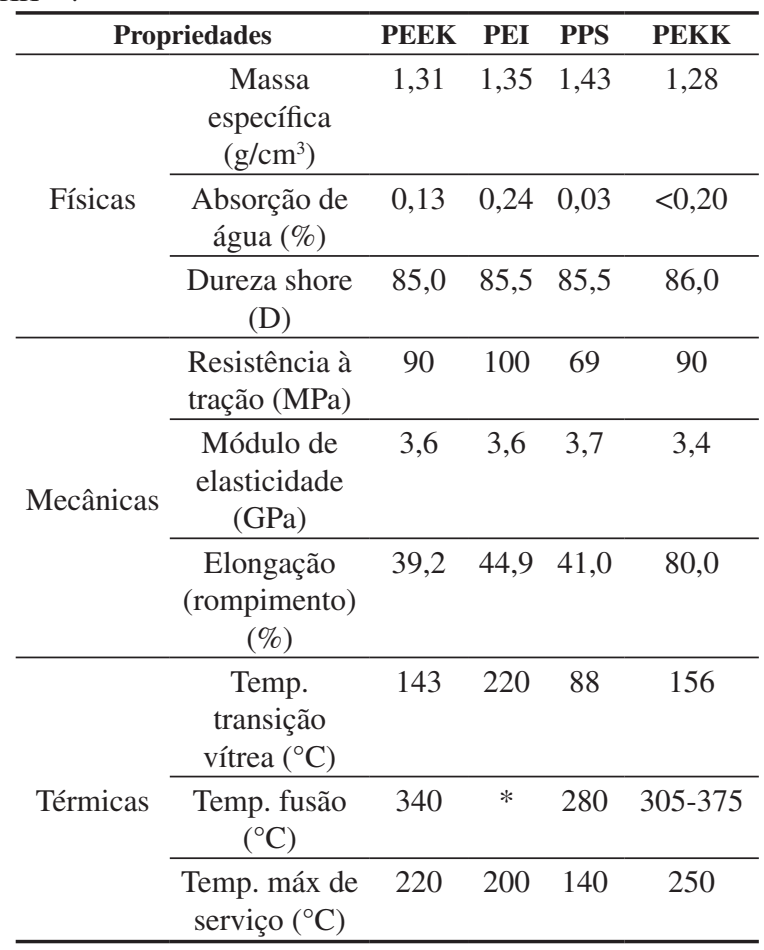

*Polímero PEI - amorfo.

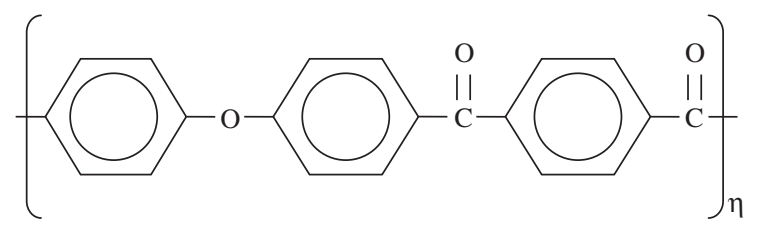

Figura 1. Estrutura química do PEKK (poli (éter cetona cetona)) $)^{[7,8]}$.

excelentes resistências mecânica, química e térmica e são diferenciados pela proporção de grupamentos éter e cetona ${ }^{[8,9]}$. O PEKK é um polímero composto de uma série de anéis aromáticos que se alternam entre uma ligação éter (-O-) e duas cetona $(\mathrm{C}=\mathrm{O})$, conforme ilustra a estrutura química na Figura $1^{[7-9]}$.

Do ponto de vista molecular, as ligações do tipo cetona são mais fortes, quando comparadas às ligações do tipo éter. Portanto, um maior número de ligações cetona contribui para o aumento da viscosidade e das temperaturas de transição vítrea $\left(\mathrm{T}_{\mathrm{g}}\right)$ e de fusão $\left(\mathrm{T}_{\mathrm{m}}\right)$. Por exemplo, o PEEK apresenta aproximadamente uma $\mathrm{T}_{\mathrm{g}}=143{ }^{\circ} \mathrm{C}$ e $\mathrm{T}_{\mathrm{m}}=340{ }^{\circ} \mathrm{C}$; o PEK

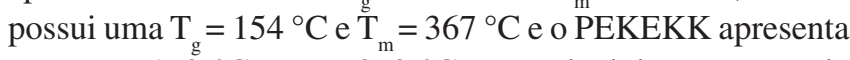
uma $\mathrm{T}_{\mathrm{g}}=173^{\circ} \mathrm{C}$ e $\mathrm{T}_{\mathrm{m}}=370^{\circ} \mathrm{C}$. Em principio, se esperaria que o PEKK apresentasse temperaturas de transição vítrea e de fusão mais elevadas, quando comparadas aos valores encontrados para o PEEK, o PEK e o PEKEKK, entretanto, a adição de grupos meta isômeros na cadeia polimérica do PEEK promove uma redução significativa da temperatura de fusão e da sua viscosidade; enquanto que, a temperatura de transição vítrea é mantida constante ${ }^{[9]}$. De acordo com a literatura ${ }^{[3-11]}$, o PEKK apresenta uma temperatura de transição vítrea de, aproximadamente, $156{ }^{\circ} \mathrm{C}$ e de fusão em torno de 305 a $310^{\circ} \mathrm{C}$, podendo alcançar valores mais elevados, segundo alguns autores ${ }^{[3,4,9]}$.

Desta maneira, para se otimizar as propriedades mecânicas de componentes fabricados a partir do PEKK, via moldagem por compressão a quente, faz-se necessário um melhor entendimento dos principais eventos térmicos que ocorrem na estrutura da matriz durante o processamento dos laminados. Para se atingir tal objetivo, o conhecimento de parâmetros do processo, tais como: temperaturas de transição vítrea $\left(\mathrm{T}_{\mathrm{g}}\right)$, de fusão $\left(\mathrm{T}_{\mathrm{m}}\right)$ e de degradação (TG) se faz necessário, pois tais propriedades podem alterar a morfologia do laminado afetando, conseqüentemente, as propriedades mecânicas do compósito final ${ }^{[10,11]}$.

O presente trabalho tem como objetivo as caracterizações térmica e reológica do polímero termoplástico PEKK, na forma semicristalina e amorfa, por meio do uso das técnicas de espectrofotometria na região do infravermelho com transformada de fourier (FTIR), calorimetria exploratória diferencial (DSC), termogravimetria (TG) e reológica. A partir dos resultados obtidos foi possível estabelecer um conjunto de parâmetros térmicos e reológicos mais apropriado a ser utilizado em um ciclo térmico de processamento durante a moldagem por compressão a quente dos compósitos termoplásticos avançados em estudo. 


\section{Experimental}

\section{Materiais}

Neste trabalho foram utilizados dois tipos de amostras do polímero termoplástico PEKK, na forma de grânulos. A primeira amostra de PEKK, denominada de semicristalina, de coloração branca, foi fornecida pela empresa Du Pont e a segunda, denominada de amorfa, de coloração marrom.

\section{Análise de absorção na região do infravermelho}

As análises espectrométricas na região do infravermelho foram realizadas utilizando-se um espectrofotômetro SPECTRUM ONE - Perkin Elmer, disponível na Divisão de Química do IAE/CTA. As condições experimentais utilizadas nestas análises foram: região de $4000-400 \mathrm{~cm}^{-1}$, resolução de $4 \mathrm{~cm}^{-1}$, ganho 1 a 20 varreduras, segundo o procedimento interno de operação PI/CIN-121 (1/0). As análises de FTIR das amostras de PEKK foram realizadas pelo uso de pastilhas de brometo de potássio, e, também, pelo uso de filmes líquidos do pirolisado das amostras, com auxílio de uma lamínula.

\section{Análise por calorimetria exploratória diferencial}

As análises por DSC foram realizadas em um equipamento Perkin Elmer Modelo Pyris 1, disponível na Divisão de Materiais do IAE/CTA, calibrado com índio e zinco, com fluxo constante de nitrogênio $(20 \mathrm{~mL} / \mathrm{min})$ e razões de aquecimento e de resfriamento de $10{ }^{\circ} \mathrm{C} / \mathrm{min}$. As amostras de PEKK, ambas com aproximadamente $6,7 \mathrm{mg}$, foram encapsuladas em um cadinho de alumínio, onde as variáveis relacionadas com as temperaturas de transição vítrea $\left(\mathrm{T}_{\mathrm{g}}\right)$, de cristalização $\left(T_{c}\right)$ e de fusão $\left(T_{m}\right)$ foram obtidas a partir de varreduras dinâmicas em uma faixa de temperaturas entre 30 a $350{ }^{\circ} \mathrm{C}$.

\section{Análise termogravimétrica}

A análise termogravimétrica foi realizada em um equipamento Perkin Elmer, TGA7 HT, disponível na Divisão de Materiais do IAE/CTA. As amostras do PEKK, ambas com aproximadamente $17,0 \mathrm{mg}$, foram ensaiadas em duas condições distintas: uma sob um fluxo constante de ar sintético e outra em nitrogênio (20 mL/min). Em ambos os casos, as amostras foram aquecidas à razão de $10{ }^{\circ} \mathrm{C} / \mathrm{min}$, na faixa de temperaturas entre 30 a $1000{ }^{\circ} \mathrm{C}$.

\section{Análise reológica}

As análises reológicas de ambas as amostras do polímero PEKK foram realizadas em um reômetro de tensão controlada da Rheometric Scientific, modelo SR-5, com pratos paralelos, disponível na Divisão de Materiais do IAE/CTA. Os ensaios foram realizados à freqüência constante de $1 \mathrm{rad} / \mathrm{s}$ e tensão de $200 \mathrm{~Pa}$, em isotermas de $330,340,345$ e $347^{\circ} \mathrm{C}$, sob atmosfera de nitrogênio. Durante o ensaio foi utilizada uma distância fixa entre os pratos superior e inferior de $1 \mathrm{~mm}$.

\section{Resultados e Discussão}

\section{Espectrofotometria na região do infravermelho com transformada de Fourier}

As amostras do PEKK, semicristalina e amorfa, foram inicialmente analisadas como recebidas utilizando-se a técnica de pastilha de $\mathrm{KBr}$. Os espectros obtidos não apresentam diferenças significativas entre as amostras, conforme mostra a Figura 2. De acordo com a literatura ${ }^{[12]}$, as principais absorções observadas encontram-se em: $3436 \mathrm{~cm}^{-1}$ (estiramento (v) do grupo $\mathrm{OH}$ ); $3064 \mathrm{~cm}^{-1}$ (grupo $\mathrm{CH}$ aromático); $1655 \mathrm{~cm}^{-1}\left(\mathrm{vc}=\mathrm{O}\right.$ de cetona conjugada); 1586 e $1494 \mathrm{~cm}^{-1}$ ( vc = c aromático); 1307, 1238, 1160 e $1111 \mathrm{~cm}^{-1}$ (vC-O do éter aromático); 842, 746 e $615 \mathrm{~cm}^{-1}$ (deformação $(\delta)$ de grupos $\mathrm{CH}$ aromáticos). Quando preparadas a partir da pirólise em bico de Bunsen, as amostras do PEKK (Figura 2 espectros c e d) apresentam, basicamente, o mesmo conjunto de absorções, acrescido de um aumento de intensidade na absorção em torno de 3365-3354 cm-1 (grupo OH) e $1694 \mathrm{~cm}^{-1}$ $(\mathrm{C}=\mathrm{O})$, devido, provavelmente, aos novos produtos de decomposição.

A partir dos resultados encontrados conclui-se que ambas as amostras de PEKK caracterizadas (amorfa e semicristalina) apresentam estruturas químicas similares, provenientes de éteres e cetonas conjugadas.

\section{Calorimetria exploratória diferencial}

A Figura 3 apresenta o resultado da análise térmica realizada por calorimetria exploratória diferencial do PEKK semicristalino. Como pode ser observado, esta amostra de PEKK apresenta uma temperatura de transição vítrea de, aproximadamente, $156{ }^{\circ} \mathrm{C}$. Nesta mesma curva observa-se, ainda, a presença de um pico endotérmico em $31{ }^{\circ} \mathrm{C}$, com início em $288^{\circ} \mathrm{C}$ e final em $325^{\circ} \mathrm{C}$, comportamento carac-

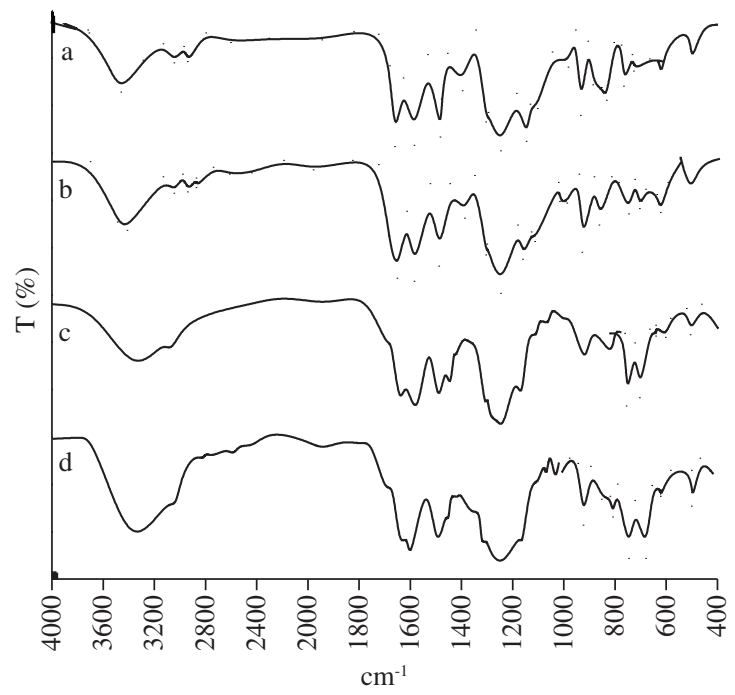

Figura 2. Espectros FT-IR de: a) amostra 1 (PEKK-semicristalino); b) amostra 2 (PEKK-amorfo); c) pirolisado - amostra 1 (PEKK- semicristalino); e d) pirolisado - amostra 2 (PEKK - amorfo $)^{[12]}$. 


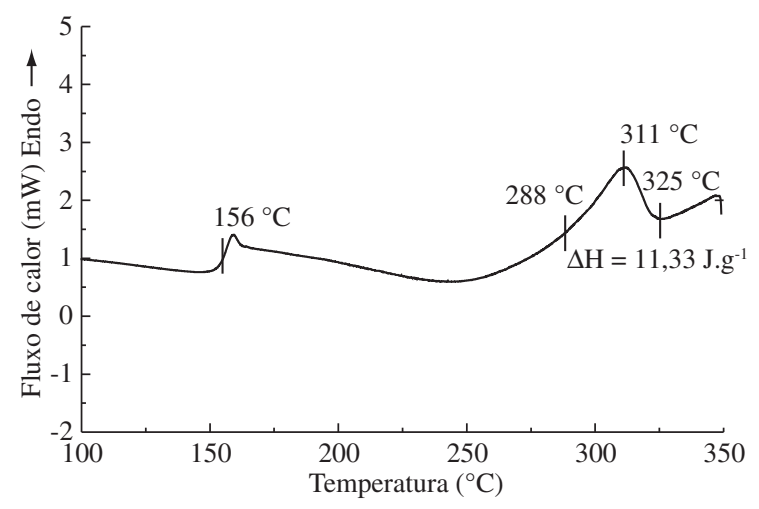

Figura 3. Curva de DSC da amostra 1 (PEKK-semicristalino) em atmosfera de nitrogênio.

terístico de polímeros semicristalinos, ou seja, a presença de um pico mais largo correspondente à fusão, ao invés de picos estreitos que ocorrem em materiais altamente cristalinos. A partir da curva apresentada foi calculada a entalpia de fusão cristalina para este polímero como sendo igual a $11,33{\mathrm{~J} . \mathrm{g}^{-1}}^{-}$.

Durante o resfriamento do PEKK semicristalino, conforme ilustrado na Figura 4, foi evidenciada a presença de um pico exotérmico em $249^{\circ} \mathrm{C}$, com inicio em $274{ }^{\circ} \mathrm{C}$ e término em $215{ }^{\circ} \mathrm{C}$. A entalpia de cristalização determinada a partir da área contida sob o pico exotérmico foi de, aproximada-

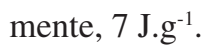

A Figura 5 apresenta o resultado da análise térmica realizada por calorimetria exploratória diferencial do PEKK amorfo. Conforme pode ser observado, o PEKK apresenta ao longo da curva uma temperatura de transição vítrea de $153{ }^{\circ} \mathrm{C}$, como também, a presença de um pico endotérmico em $300{ }^{\circ} \mathrm{C}$, com início em $288^{\circ} \mathrm{C}$ e final em $310^{\circ} \mathrm{C}$. A entalpia, determinada pela área contida sob o pico endotérmico, é de aproximadamente $2{\mathrm{~J} . \mathrm{g}^{-1}}^{-1}$ Tanto os valores de temperatura de fusão, como os de entalpia, são inferiores aos determinados para o PEKK denominado de semicristalino. Este fato, somado à observação que durante o resfriamento não foi observado nenhum pico exotérmico, que caracterizaria o

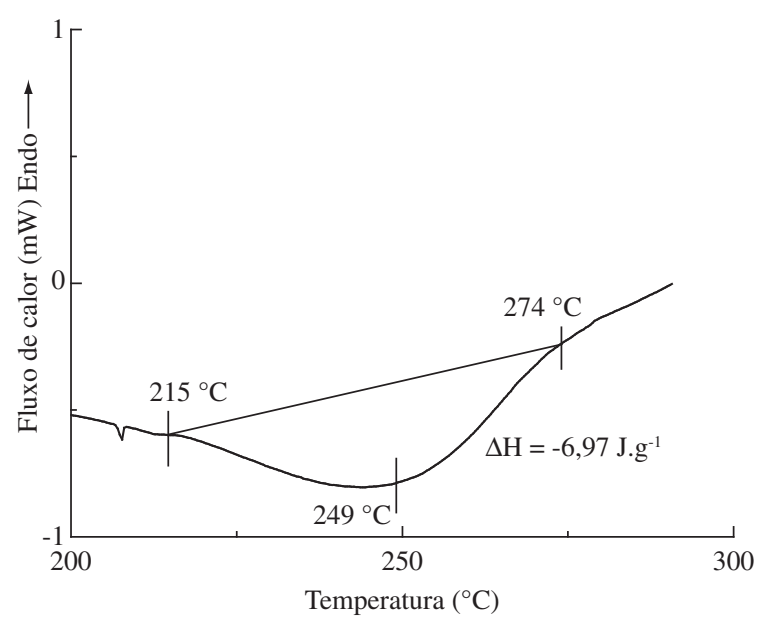

Figura 4. Pico exotérmico observado durante o resfriamento da amostra 1 (PEKK-semicristalino) em atmosfera de nitrogênio.

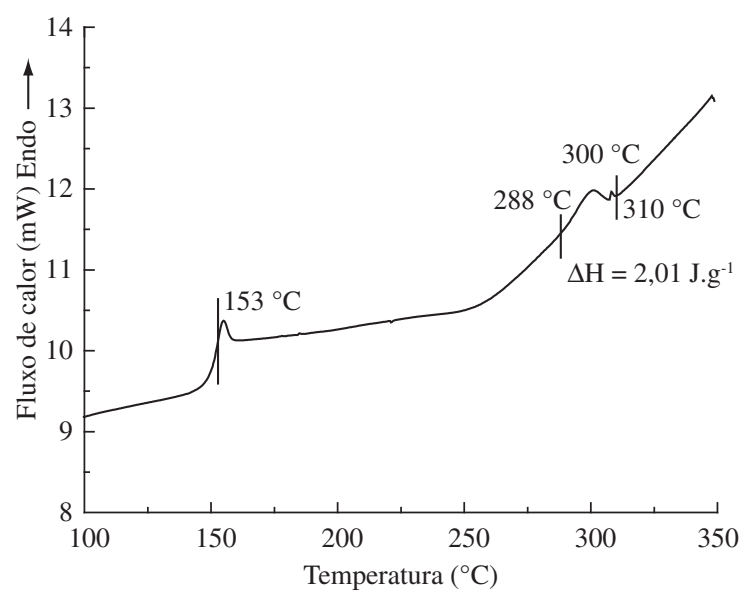

Figura 5. Curva de DSC da amostra 2 (PEKK-amorfo) em atmosfera de nitrogênio.

processo de cristalização do material, indica que nesta matriz predomina a morfologia amorfa.

Os resultados das temperaturas de transição vítrea $\left(\mathrm{T}_{\mathrm{g}}=156{ }^{\circ} \mathrm{C}\right)$ e de fusão cristalina $\left(\mathrm{T}_{\mathrm{m}=} 31{ }^{\circ} \mathrm{C}\right)$ do PEKK semicristalino estão concordantes com valores encontrados na literatura ${ }^{[3-11]}$, entre estes, os disponibilizados por Bai, Leach e Pratte (2004). Porém, o PEKK amorfo exibe valores de temperaturas de transição vítrea de $153{ }^{\circ} \mathrm{C}$ e de fusão igual a $300{ }^{\circ} \mathrm{C}$, respectivamente, resultados esses ligeiramente inferiores ao do material semicristalino. Este fato está provavelmente relacionado com o caráter predominantemente amorfo que o PEKK analisado apresenta e, também, a uma provável mais baixa distribuição de massa molar, quando comparado aos dados disponibilizados para outras amostras de PEKK encontradas na literatura ${ }^{[3-11]}$.

As análises de ambas as amostras do PEKK, semicristalina e amorfa, também confirmam que existe uma redução significativa da temperatura de fusão $\left(\mathrm{T}_{\mathrm{m}}\right)$, quando comparadas aos polímeros de sua família PAEK (PEEK, PEK, PEKEKK), fato este que pode estar relacionado com a adição de grupamentos meta isômeros, conforme citado na literatura ${ }^{[9]}$.

A obtenção dos resultados deste estudo contribuiu significativamente para a caracterização dos principais parâmetros térmicos das amostras do polímero termoplástico PEKK, semicristalino e amorfo, que serão utilizadas na fabricação de laminados com fibras de carbono, via moldagem por compressão a quente.

\section{Análise termogravimétrica}

A Figura 6 apresenta as curvas termogravimétricas do PEKK semicristalino sob as atmosferas de ar sintético e de nitrogênio $\left(\mathrm{N}_{2}\right)$. Conforme pode ser observado, quando a amostra é ensaiada sob atmosfera de nitrogênio, esta apresenta uma temperatura inicial de degradação de $403{ }^{\circ} \mathrm{C}$. Já, quando analisada sob atmosfera de ar sintético, o polímero apresenta uma redução da temperatura de início de degradação, que ocorre em $366^{\circ} \mathrm{C}$, como esperado, devido à oxidação do material. 


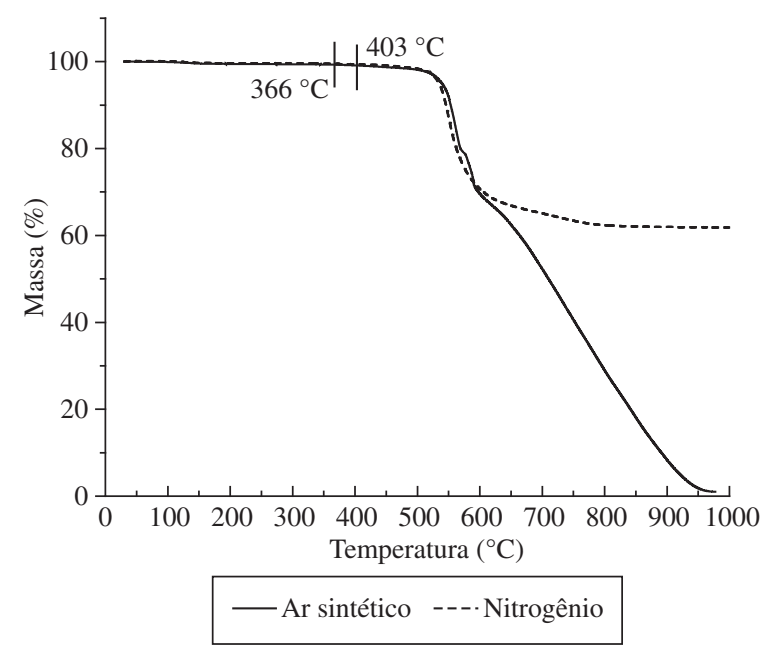

Figura 6. Análise termogravimétrica da amostra 1 (PEKK-semicristalino) sob atmosfera de ar sintético e nitrogênio.

A Figura 7 apresenta as curvas termogravimétricas do PEKK amorfo sob as atmosferas de ar sintético e de nitrogênio. Conforme pode ser observado, quando ensaiada sob atmosfera de nitrogênio, esta amostra apresenta uma temperatura de início de degradação em $364^{\circ} \mathrm{C}$. Entretanto, quando ensaiada sob atmosfera de ar sintético esta mostra uma redução da temperatura inicial de degradação para $352^{\circ} \mathrm{C}$.

Apesar das temperaturas de início de degradação térmica de ambas as amostras apresentarem-se diferentes, estas mostram comportamentos similares de perda de massa, ou seja, constata-se uma perda de massa de, aproximadamente, $30 \%$, entre as temperaturas de 500 e $600{ }^{\circ} \mathrm{C}$, devido à degradação da matriz polimérica, para ambos os tratamentos (ar sintético e $\mathrm{N}_{2}$ ). Observa-se, também, em ambos os materiais, uma pequena perda de massa antes da temperatura de início de degradação. Este comportamento está, provavelmente, relacionado às presenças de umidade $(<0,2 \%)$ e de voláteis $(<0,7 \%)$, de acordo com as informações dos fabricantes ${ }^{[3,4]}$. Já, quando analisadas entre as temperaturas de 600 e $1000{ }^{\circ} \mathrm{C}$, sob fluxo de nitrogênio, as amostras 1 e 2 apresentam uma perda de

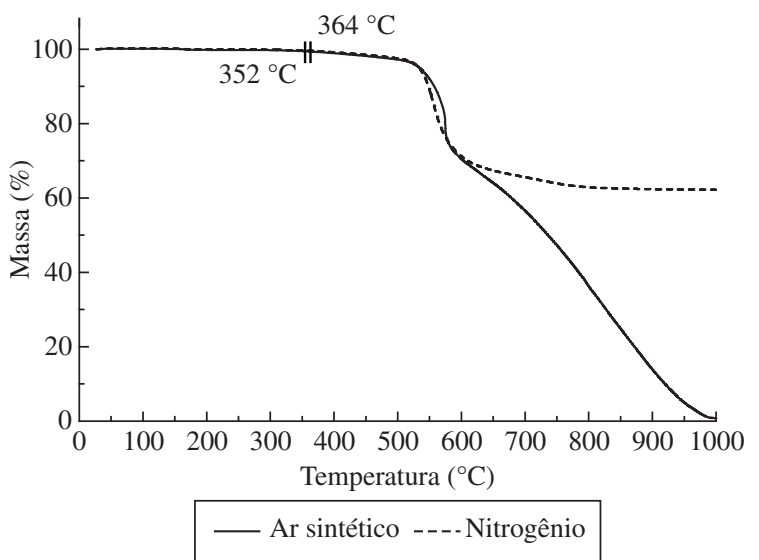

Figura 7. Análise termogravimétrica da amostra 2 (PEKK-amorfo) sob atmosfera de ar sintético e nitrogênio. massa de, aproximadamente, $8 \%$, resultando em um teor de cinzas e de carbono fixo em torno de $62 \%$. Quando as amostras são analisadas até $1000^{\circ} \mathrm{C}$, sob atmosfera oxidante, praticamente toda a matriz polimérica se degrada. A partir destas análises foi possível determinar as temperaturas máximas de processamento dos laminados, que poderão ser utilizadas durante o ciclo de moldagem por compressão a quente, sem que ocorra a degradação da matriz polimérica.

\section{Análise reológica}

As Figuras 8 e 9 mostram a variação da viscosidade complexa $(\eta *)$ em função do tempo, sob condições isotérmicas, das amostras de PEKK semicristalino e amorfo, respectivamente. Como esperado nas análises isotérmicas, à medida que se aumenta à temperatura do ensaio, observa-se que ocorre uma redução do valor da viscosidade. Para o PEKK semicristalino, quando analisado em isotermas de 340,345 e $347^{\circ} \mathrm{C}$, observa-se que a viscosidade praticamente não varia em função do tempo, considerando-se que o material em análise é mantido sob atmosfera inerte. Entretanto, o PEKK amorfo, quando analisado sob a temperatura de $330{ }^{\circ} \mathrm{C}$, apresen-

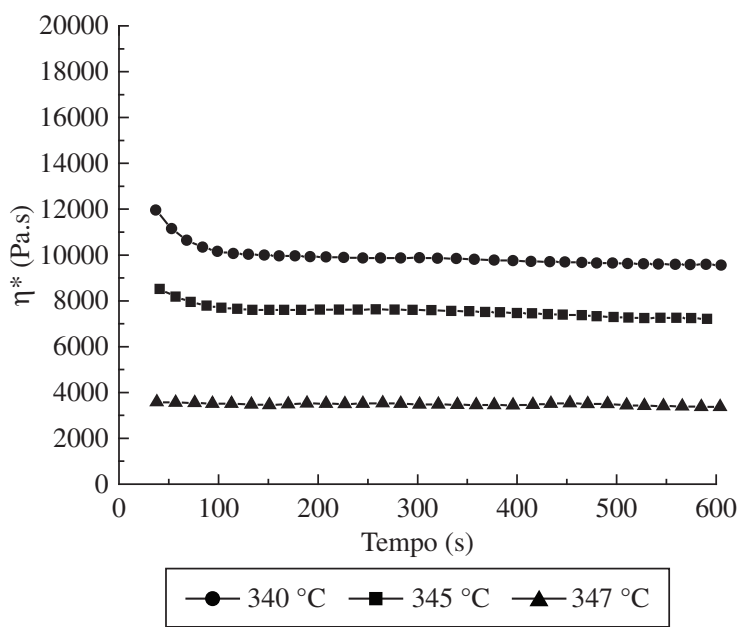

Figura 8. Resultado da análise reológica da amostra de PEKK semicristalino.

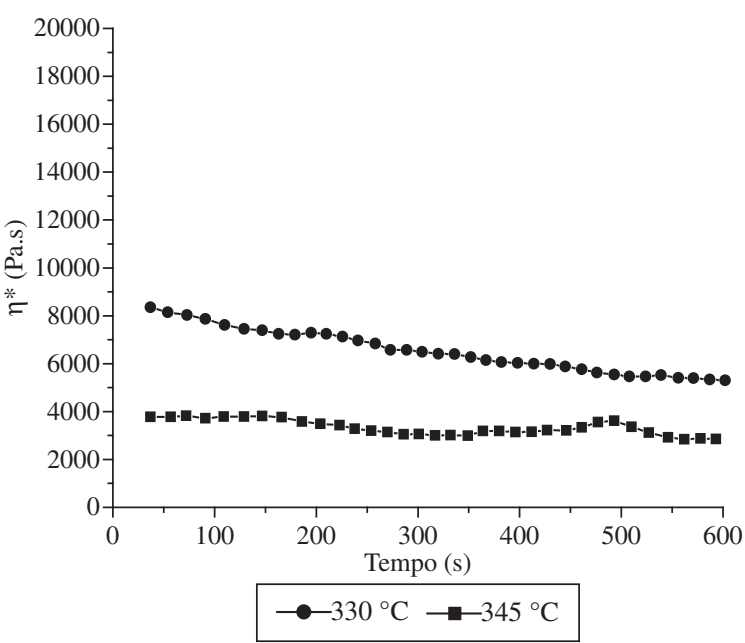

Figura 9. Resultado da análise reológica da amostra de PEKK amorfo. 
ta uma redução no valor da viscosidade complexa $(\eta *)$ de, aproximadamente, $30 \%$ durante o mesmo tempo de análise (10 minutos). Neste caso, observa-se, ainda que, na temperatura de $345{ }^{\circ} \mathrm{C}$ ocorrem algumas irregularidades nos valores de viscosidade complexa, sugerindo uma menor estabilidade térmica do polímero.

Como pode ser observado na Figura 8, o valor encontrado para a viscosidade complexa $(\eta *)$ a $347{ }^{\circ} \mathrm{C}$ é aproximadamente $65 \%$ menor quando comparado ao valor encontrado para $340{ }^{\circ} \mathrm{C}$. Um comportamento análogo é observado na Figura 9 , onde o valor da viscosidade complexa a $345^{\circ} \mathrm{C}$ é praticamente $50 \%$ menor do que o valor encontrado a $330^{\circ} \mathrm{C}$, ou seja, a utilização de temperaturas mais elevadas contribui significativamente para a diminuição da viscosidade complexa $(\eta *)$. De acordo com a literatura ${ }^{[13-15]}$, este comportamento é atribuído à destruição gradativa das forças de interação existentes entre as macromoléculas (ligações secundárias), associadas ao volume livre da matriz. Este decréscimo da viscosidade do polímero é desejável, pois favorece a percolação e a distribuição da matriz polimérica fundida no reforço. Esta estruturação pode também ser destruída com o aumento da taxa de cisalhamento e facilmente recomposta quando este esforço cisalhante diminui.

A correlação destes resultados com os obtidos nas análises termogravimétricas permitiu estabelecer as temperaturas mais adequadas para a consolidação dos laminados os valores de $350{ }^{\circ} \mathrm{C}$ para o PEKK semicristalino e de $345^{\circ} \mathrm{C}$ para o PEKK amorfo. Vale ressaltar que, temperaturas inferiores a estas promovem valores de viscosidades inadequados para a impregnação da matriz no reforço e temperaturas mais elevadas se aproximam das temperaturas de degradação das matrizes poliméricas em estudo. Nestas temperaturas de processamento, o PEKK apresenta viscosidades complexas ( $\eta *$ ) entre 3000 a 4000 (Pa.s) para ambas amostras, valores estes superiores aos obtidos em literatura ${ }^{[9,15-16]}$, entretanto, este fato pode estar provavelmente relacionado com aplicação de temperaturas mais elevadas $\left(\sim 400^{\circ} \mathrm{C}\right)$ durante o ensaio. Estes resultados de viscosidade para o PEKK viabilizam a obtenção de um ciclo térmico adequado para a moldagem por compressão a quente, com temperaturas de processamento entre a fusão e a degradação, ciclo este que favorece a percolação e a distribuição da matriz polimérica fundida no reforço.

\section{Proposição do ciclo térmico de processamento}

Devido à semelhança dos resultados encontrados tanto para a matriz de PEKK semicristalina quanto para a amorfa, neste trabalho foi estabelecido um ciclo de processamento idêntico para ambas as amostras.

$\mathrm{O}$ ciclo térmico de processamento proposto, conforme pode ser observado na Figura 10, divide-se em três fases: fusão, consolidação e solidificação, iniciando-se com a elevação da temperatura a partir da ambiente $\left(\sim 25^{\circ} \mathrm{C}\right)$ até a sua temperatura de consolidação $\left(\mathrm{T}_{\mathrm{Con}}\right)$, sendo estas obtidas a partir dos ensaios de reologia, discutidos anteriormente. Durante a primeira etapa do processo de moldagem por

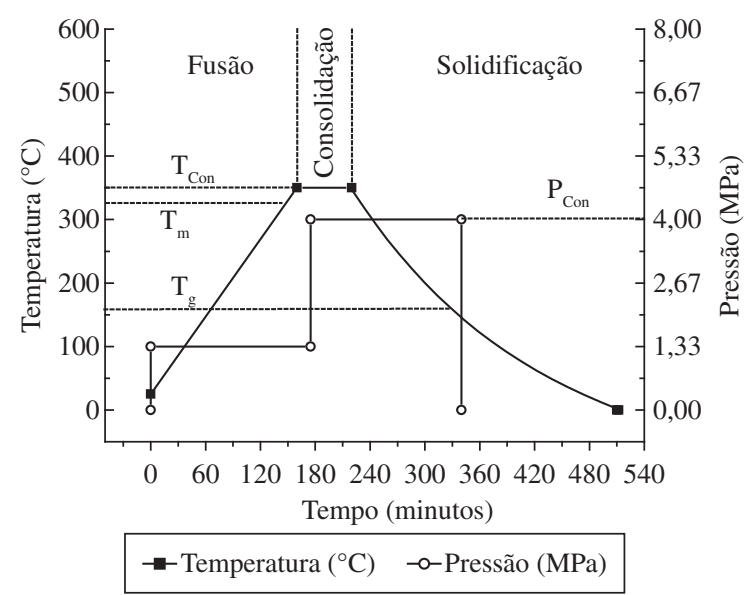

Figura 10. Proposição do ciclo térmico para a moldagem por compressão à quente do compósito PEKK/fibra de carbono contínua $\left(\mathrm{T}_{\text {Con }}=\right.$ Temp. de Consolidação; $\mathrm{T}_{\mathrm{m}}=$ Temp. de Fusão; $\mathrm{T}_{\mathrm{g}}=$ Temp. de Transição Vítrea; $\mathrm{e}$ $\mathrm{P}_{\text {Con }}=$ Pressão de Consolidação).

compressão a quente (fusão), as camadas de fibra de carbono (60\% em volume) e PEKK ( $40 \%$ em volume) foram compactadas utilizando-se a pressão de, aproximadamente, 1,3 MPa. Esta etapa objetiva uma transferência de calor homogênea para todo o laminado. Alcançada a temperatura de consolidação $\left(350{ }^{\circ} \mathrm{C}\right.$ para a matriz semicristalina e $345^{\circ} \mathrm{C}$ para a amorfa), fez-se um patamar por 15 minutos, ao final do qual foi dado início à segunda etapa do ciclo de moldagem, que se refere à consolidação do laminado. Nesta fase, a pressão de compactação do laminado foi elevada para $4 \mathrm{MPa}$, com um patamar de 1 hora nesta condição, de modo a garantir a impregnação homogênea do reforço. Tanto a primeira, quanto a segunda etapa, foram baseadas nos resultados obtidos nos ensaios reológicos e nas análises térmicas, como discutido anteriormente. A terceira etapa do processo de moldagem por compressão a quente envolveu o resfriamento natural do ferramental de moldagem, ainda sob pressão.

A partir da utilização deste ciclo de processamento, amostras de compósitos de PEKK/fibras de carbono foram obtidas e as avaliações mecânica e físico-químicas destes compósitos vêm sendo realizadas com o intuito de utilizar este material em aplicações primárias e secundárias de aeronaves nacionais.

\section{Conclusão}

A partir dos resultados obtidos via análises térmicas e reológicas foi possível propor um ciclo térmico a ser utilizado no processo de moldagem por compressão a quente de laminados termoplásticos de PEKK com fibras de carbono contínuas. Duas amostras do polímero termoplástico PEKK, uma denominada de semicristalina e a outra de amorfa, foram caracterizadas, visando o processamento de compósitos poliméricos avançados. Análises de FTIR não mostraram diferenças estruturais significativas entre as duas amostras analisadas, observando-se, de maneira geral, que ambos po- 
límeros são constituídos essencialmente de grupamentos aromáticos tipos éter e cetona, como esperado.

Ambas as amostras do PEKK (semicristalina e amorfa) apresentaram temperaturas elevadas de transição vítrea $\left(\mathrm{T}_{\mathrm{g}}=156 \mathrm{e} 153{ }^{\circ} \mathrm{C}\right.$, respectivamente). Estas temperaturas mostram-se adequadas para o processamento de artefatos que exigem elevadas temperaturas de serviço em suas aplicações finais. Temperaturas de fusão moderadas $\left(\mathrm{T}_{\mathrm{m}}=325\right.$ e $310^{\circ} \mathrm{C}$, respectivamente) foram determinadas. Esta característica mostra-se adequada, pois reduz o custo de processamento e, conseqüentemente, melhora a competitividade de uso em relação aos outros polímeros dessa família PAEK que vem sendo atualmente mais utilizados em aplicações aeroespaciais.

Definiu-se como as temperaturas mais adequadas para a consolidação dos laminados, obtidas a partir da correlação entre as análises reológica e termogravimétrica, as temperaturas de $350{ }^{\circ} \mathrm{C}$ para a o PEKK semicristalino e de $345{ }^{\circ} \mathrm{C}$ para o PEKK amorfo, e a faixa de viscosidade complexa $(\eta *)$ entre 3000 a 4000 (Pa.s), para ambas amostras de PEKK.

A partir deste trabalho, pretende-se contribuir para o estudo da correlação de parâmetros reológicos e térmicos no estabelecimento de metodologias adequadas ao processamento de compósitos poliméricos termoplásticos para aplicações aeroespaciais.

\section{Agradecimentos}

Os autores agradecem à Divisão de Materiais do IAE/CTA, em particular à técnica Andreza Moura pelas análises térmicas realizadas, a empresa Du Pont pelo fornecimento das amostras de PEKK e à Divisão de Química do IAE/CTA pela realização das análises de FTIR, assim como à FAPESP e à CAPES pelo suporte financeiro.

\section{Referências Bibliográficas}

1. Daniel, I. M. \& Ishai, O. - "Engineering Mechanics of Composites Materials", Oxford University Press, New York (1994).

2. Burakowiski, L. - "Estudo da Interface de Compósitos Termoplásticos Estruturais Processados a Partir de Fibras de Carbono com Superfícies Modificadas", Tese de Mestrado, Instituto Tecnológico de Aeronáutica, São José dos Campos - SP, Brasil (2001).

3. Material de informação do PEKK. CYPEK ${ }^{\mathrm{TM}}$ Polyetherketoneketone (PEKK) Thermoplastic Resin (1999).
4. Material Identification PEKK Resin Gradem F, MSDS Number: FML027, Du Pont (1992).

5. Bai, J.; Leach, D. \& Pratte J. - "Properties, Processing and Fabrication of Poly (Ether-Ketone-Ketone) Composites", Sampe Europe Conference (2004).

6. Mazur, R. L.; Costa, M. L.; Botelho, E. C. \& Rezende, M. C. - Estudo do Comportamento Térmico da Matriz Termoplástica PEKK Utilizada na Indústria Aeronáutica. $17^{\circ}$ Congresso Brasileiro de Engenharia e Ciência dos Materiais, Foz do Iguaçu, PR, Brasil.

7. Mckague, L. - "Thermoplastic Resins”, in: ASM Handbook, 21, Composites, ASM International, Materials Park, OH, 44073-0002, ISBN: 0-87170-703-9, (2006).

8. Lee, S. M. - "Encyclopedia of Composites", 6, Stuar M. Lee, VCH, New York (1989).

9. Hsiao, B. S.; Chang I. Y. \& Sauer B. B. - Polymer, 32, p.2799-05, (1991).

10. Hojjati, M.; Chouinard, G. \& Yousefpour, A. - "Crystallization Behavior of PEKK Thermoplastic Polymer", Aerospace Manufacturing Technology Center, Institute for Aerspace Research, National Research Council Canada, Montreal, Quebec H3T 2B2, Canada.

11. Salek, M. H. - "Effects of Processing Parameters on the Mechanical Properties of Carbon/PEKK Thermoplastic Composite Materials", Master of Applied Science at Concordia University Montreal, Quebec, Canada, (2005).

12. Hummel, D. O. \& Scholl, F. - "Atlas of polymer and plastics analysis" Verlag Chemie GmbH, D6940 Weinhein, 1 (espectro 1864), (1984).

13. Botelho, E. C. - "Compósitos Aeronáuticos Processados a Partir de Fibra de Carbono Impregnadas com Poliamida 6/6 Via processo de Polimerização Interfacial", Tese de Doutorado, Instituto Tecnológico de Aeronáutica (ITA), São José dos Campos - SP, Brasil (2002).

14. Young, D. G. \& Baird, D. G. - Composites, Part B: engineering, 31, p.209-21, (2000).

15. Bretas, R. E. S. \& D'Ávila, M. A. - "Reologia de Polímeros Fundidos", $2^{a}$ ed., edufscar, São Carlos, (2005).

16. Hopper, L. C \& Sauer, G. L. - "Properties Test for Matrix Resins" in:Composites - Engineered Materials Handbook, 1, ASM International, Materials Park, OH, 44077-0002, ISBN: 0-87170-279-7, 1, (1993).

Enviado: $20 / 12 / 07$

Reenviado: 09/04/08

Aceito: 05/05/08 